\title{
Investigation of commuting and non-commuting travel features for the popularization of public transportation system
}

\author{
Ye Sun ${ }^{1,}$ Xinmiao Yang \\ Department of Civil Engineering, Tsinghua University, \\ Beijing 100084, PR China \\ Received 20 August 2011 \\ Accepted 25 November 2011
}

\begin{abstract}
The increasing travel demands and the growing diversity of trip purposes in megacities are placing more pressure on the urban transportation system, and there is an urgent need to popularize public transportation and control the exploding increase in travels by private cars. In order to enhance the competence of public transportation and establish a balance among various travel modes in megacities, as a highlight example, Beijing residents' commuting and non-commuting travel features has been investigated focusing on the number of trips per capita per day, trip purposes, travel modes and proportion of commuting and non-commuting travels in public transportation. To better understand the current transportation situation and the changes of travel patterns in Beijing, corresponding data of New York, Hong Kong and London are comparatively studied. It is found that the per capita number of trips in Beijing has dramatically increased, and comparative analyses with the most developed cities shows that in metropolises with mature transportation system, commuting-and non-commuting travel relies a great deal on public transportation. It is recommended that the improvement of the public transportation system should be put into top priority in such megacities intelligent transportation planning and development in order to satisfy the increasing travel demands of Beijing residents, especially non-commuting demands that have long been overlooked.
\end{abstract}

Key words: non-commuting travel; intelligent transportation system; comparison investigation

\section{Introduction}

With the ever-accelerated development of science and technology in China during the past few decades, great changes have taken place in various aspects of people's life, but the increasing motorized traffic also brings about inescapable challenges.

The relationship between economic growth and transport demand is a widely studied problem. It is universally acknowledged that the increasing prosperity of a city would naturally trigger demands of transportation development, and the improvement of infrastructure system would pave the way for social and economic development. In 2003, the research group of travel demand of Beijing Social Economic Research Agency (BSERA) presented a report which focused on the trend of social and economic growth from 2003 to 2020 and its relationship to transport demand. It is stated in the report that the quality of live has experienced substantial improvement, and it enables people to pursue better travel modes and conditions. In recent years, the percentage of travel expenditure of Beijing residents climbed up continuously. Meanwhile, the establishment of social insurance system and the improvement of credit business give birth to the concept of excessive consumption. Therefore, people are more generous to purchase more convenient and comfortable travel modes, which can be substantiated by the exploding of private car ownership ${ }^{1}$.

*Corresponding author: sunye08@mails.tsinghua.edu.cn 
The increasing amount of car ownership reflects the prosperity of Beijing and the improvement of people's economic conditions on a large scale, but it would inevitably bring about serious pressure on the road system. It is a notorious phenomenon that during the peak hours the road system in certain parts of Beijing has already reached an ultra saturated condition. And it is not a rare scene that vehicles line up at traffic lights for as long as a block waiting to cross the road, but only several cars can get through before the lights change again. Simultaneously due to various road conditions and drivers' behaviors, the issue of traffic safety has also become a public problem². Meanwhile, the growing severity of air pollution has long plagued the society, resulting in the decline of people's health condition and exerting a negative effect on the reservation of cultural relics and historical sites ${ }^{3-4}$.

To solve these problems, the Beijing municipal government has taken a series of tentative measures. The policy of license plate restriction was put forward in 2008. Except that, a new policy, implemented from April 1st 2011, raised the rate of non-residential parking fees to a much higher level. Simultaneously, parking fees on the road have also been raised to as high as 20 yuan an hour in certain areas. These measures, along with the skyrocketing oil price, are expected to discourage Beijing residents from driving private cars.

Nevertheless, the limitations on private cars are not permanent solutions to the problems. According to the report by BSERA, one of the major reasons for Beijing residents to buy private cars is that the current public transportation system cannot satisfy their travel demand ${ }^{1}$. The planned route network can neither keep up with the pace of the expanding city nor satisfy various commuting and non-commuting travel demands. To some extent, people have no better choices but to buy private cars ${ }^{5}$. Therefore, the need for better transport systems throughout the city to cope with the increasing traffic pressure has become urgent. In current situations, the priority is to plan and develop a more comprehensive public transport system, which could satisfy the traveling needs of Beijing residents so that they would eventually consider public transport as their first choice ${ }^{6}$.

To better satisfy the travel demands of Beijing residents, it is of essential importance to study their travel features. Trips could be divided into two categories: commuting travel and non-commuting travel. Commuting travel refers to trips to/from work and school, and non-commuting travel refers to trips for shopping, recreational, social and private purposes. Commuting travel constitutes the major part of transport plans, and is relatively inflexible in origin and destination and in time of day. Meanwhile, rapid social changes and economic developments have diversified people's travel demand. The needs for non-commuting travel have experienced a substantial increase, and the trend would be kept in the coming

Table 1: Transportation surveys in Beijing

\begin{tabular}{ccc}
\hline Year & Survey feature & Region \\
\hline 1986 & The first overall survey & All regions of the city \\
2000 & The second overall survey & All regions of the city \\
2002 & Sample survey & All regions of the city \\
2004 & Sample survey & Huilongguan,Qinghe, Tiantongyua, Beiyuan \\
2005 & Sample survey & The eight urban districts and suburban counties \\
2006 & The third overall survey & All regions of the city \\
2007 & Sample survey & The eight urban districts \\
& Sample survey & The eight urban districts
\end{tabular}

Source: Annual Report of Beijing Transport Development (1986-2007) 2

years ${ }^{7}$. This paper focuses the study on the commuting and non-commuting travel features of Beijing residents, and the role public transportation plays in satisfying the commuting and non-commuting travel demands of the general public. Since non-commuting travel is a newly generated travel demand which is more flexible compared with commuting travel, and-popularization of public transportation is urgently needed in Beijing, this paper

\footnotetext{
${ }^{2}$ Unless otherwise specified, the survey data about Beijing transport features in this paper are all cited from Annual Report of Beijing Transport Development (1986-2007).
} 
aims to the proper guide for the future development of public transportation based on the features of non-commuting demand.

The data of Beijing residents' travel characteristics is analyzed, including the number of trips per capita, trip purposes, travel mode for commuting and non-commuting purposes and percentages of commuting and non-commuting travel by public transportation. As a comparison, travel features of resident's of New York, Hong Kong, and London are investigated as well. This paper is structured as follows: the next section presents detailed description of the cities investigated and data sources. It is followed by various transportation data of Beijing, New York, Hong Kong, and London regarding number of trips, trip purpose, travel modes and their distribution in commuting and non-commuting travels, and proportion of commuting and non-commuting travels in public transportation. Finally, some conclusions and future work are summarized briefly.

\section{Cities surveyed and data sources}

The data for analysis mainly comes from the residents travel characteristics survey in the past twenty years in various regions of Beijing, which is presented in "Annual Report of Beijing Transport Development" from 1986-2007 published by Beijing Transportation Research Center (TRC) $)^{8-15}$. The method and regions of the surveys conducted across the twenty year time span are not totally the same, as shown in Table 1.

The definitions of "trips" for these different surveys are discrepant. For example, in the 2005 survey, the concept of "trip" was defined based on activities. "A trip" was defined as:

- Trips with definite goals and destinations

- Trips that pass by streets or districts with a formal name

- Trips disregard of the length of time and total distance

The definition in the 2005 survey is different from those in the surveys in 1986 and 2000, and the discrepancies are illustrated in Table 2.

Table 2: The discrepancies in the definitions of "trip" in different surveys

\begin{tabular}{cc}
\hline Year of survey & Definition \\
\hline $1986 、 2000$ & Trips more than 500m taking more than 8 minutes \\
2005 & No limit to distance and time \\
\hline
\end{tabular}

Although these two definitions are slightly different, it should be justifiable that trips that take less than 8 minutes and shorter than $500 \mathrm{~m}$ are usually taken on foot. Trips on foot are not discussed in this paper. Therefore, it is reasonable to overlook the influence brought about by this discrepancy in definition.

For comparisons, three other cities, New York, London, and Hong Kong, are chosen and investigated. New York and London are the most developed metropolises in the world, and the travel patterns of residents in these cities is studied for a better understanding of the situation of transportation system in matured international cities. Hong Kong as well has established a rather complete public transportation system, and a considerable majority of Hong Kong residents regard public transportation their first choice when traveling. Studying the experience of Hong Kong would be conducive for the exploration of a reasonable and effective way to promote the concept of "public transportation oriented city” in Beijing. The investigation scales and data sources of cities are shown in Table 3.

Table 3: Investigation scales and data sources of different cities

\begin{tabular}{lll}
\hline City & Investigation scale & Data sources \\
\hline Hong Kong & The whole city & Travel Characteristics Survey 2002 Final Report $^{16}$ \\
New York & Bronx, Kings, Queens, New York, Richmond & 1995, 2001 National Household Travel Survey New York Add-On \\
London & Greater London & Transport Statistics for Metropolitan Areas (2000) \\
& & Report 2007 $^{20}$ \\
\hline
\end{tabular}




\subsection{Number of trips.}

The number of trips per day reflects people's needs for transport on a regular basis. The indicator most commonly used is the per capita times of trip, which is defined as the ratio of the total amount of trips in the scale surveyed and the number of respondents. The per capita number of trips per day for Beijing residents in 1986, 2000 and 2002 is presented in Fig.1, and Fig.2 illustrates the numbers of trips per capita per day from 2003 to 2007 annually.

Fig. 1 shows that the number of trips per capita per day experienced a striking ascent from 1986 to 2000, soaring to 2.77 in 2000 compared to 1.61 in 1986 . The figure climbed to a climax in 2002, with 2.82 times on average-of a daily basis. Fig.2 illustrates that the number from 2003-2007 reached a relative stable state despite some slight fluctuations in the range between 2.4 and 2.53. It is noticeable that in2005 the figure lightly surpass the average level from 2003 to 2007, which could be interpreted by the disparity of region surveyed between 2005 (All regions of the city) and other years (Selected sample areas).

In comparison with the figures of New York and London, it can be found that the average number of trips of Beijing is getting close to that of these two cites.

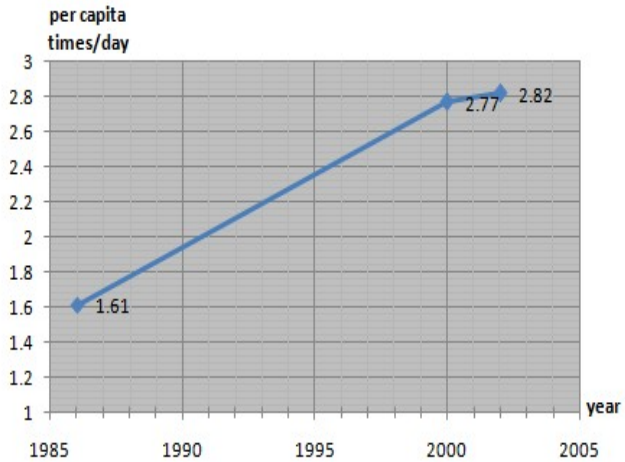

Fig. 1. Number of trips per capita per day in Beijing (1986, 2000, 2002)

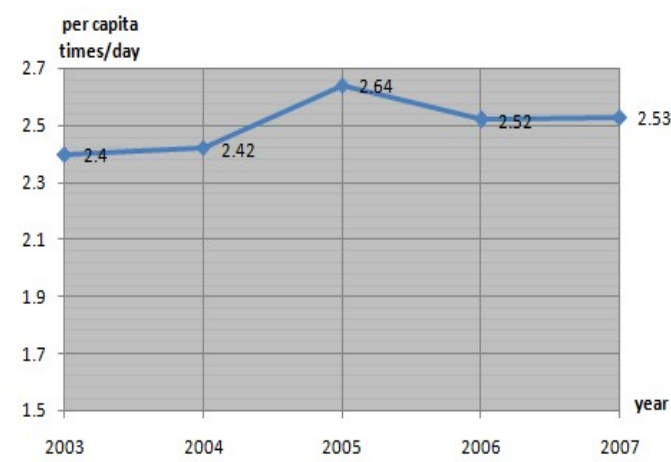

Fig. 2. Number of trips per capita per day in Beijing (2003-2007) metropolitans (See Table 4.), especially London.

Table 4: The number of trips per capita per day in New York and London （number of trips per capita/day )

\begin{tabular}{llll}
\hline & 1986 & 1995 & 2001 \\
\hline New York & - & 3.58 & 3.39 \\
London & 2.87 & 2.67 & - \\
\hline
\end{tabular}

Source: National Household Travel Survey New York Add-On (1995; 2001); Transport Statistics for Metropolitan Areas (2000)

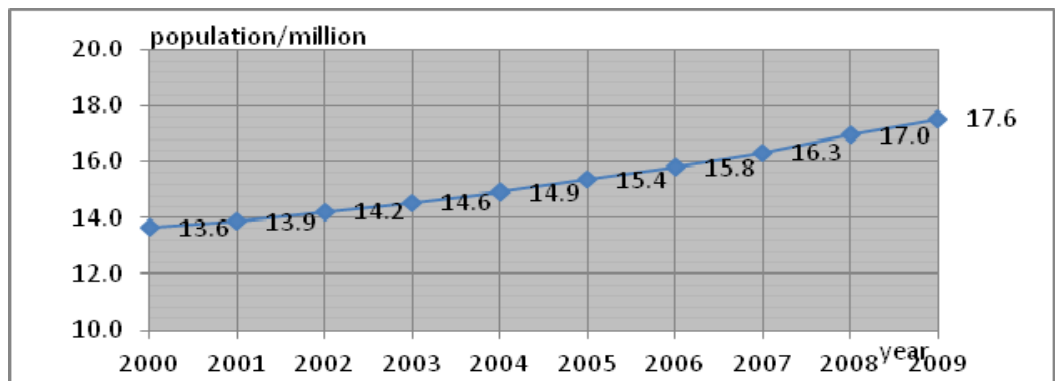

Fig. 3. Number of permanent population in Beijing (2000-2009) Source: Beijing Statistical Yearbook $(2010)^{21}$

It is illustrated that Beijing residents' need for transport has approximately swelled to the same level as that of London, and the per capita travel demand is lower than that of New York. Supposing that the number of trips per capita will stay in a fluctuating condition, the whole travel demand in Beijing is of all likelihood to increase rapidly, taking its growing population into consideration. The data of permanent population in Beijing during the past ten 
years are shown in Fig.3. Asian Development Bank pointed out in its statistic report of 1989 that when the population of a city in a developing country increased by 1 million, there would be an increase in public transportation travel demand by 350 to 400 thousand times. Consequently, it is evident that efficient transport will become one of the greatest challenges to the transportation system.

The level of economic development and geographical aspects are likely to be significant factors in determining the number of trips of Beijing residents. Families with a higher income and a private car ownership are more inclined to go out. When family income increases, various travel demand is likely to emerge. Investigations of trip purpose in next section will highlight on this problem.

\subsection{Trip Purpose}

Trip purpose refers to the motivation to travel. People take a trip for a variety of reasons, including commuting, dealing with personal and family issues, social and recreational activities. Different countries categorize trip purposes in different ways. For example, the National Personal Transportation Survey-resources by the US Federal government to study contribution of various traffic motivations to transit ridership classified trip purposes into 21 types. In China, these purposes are simplified into 8 types: commuting, schooling, trips back home, business trips, return trips, shopping, daily routines and other trips ${ }^{12}$. On the basis of different random sampling in previous surveys and investigations, the proportions of different trip purposes from 1986 to 2007 are shown in Table 5.

Table 5: Trip purpose of Beijing residents from 1986 to 2007 (\%)

\begin{tabular}{|c|c|c|c|c|c|c|c|c|}
\hline Trip Purpose & 1986 & 2000 & 2002 & 2003 & 2004 & 2005 & 2006 & 2007 \\
\hline Commuting & 30.17 & 22.69 & 18.30 & 29.34 & 26.32 & 19.07 & 21.76 & 22.24 \\
\hline Schooling & 7.87 & 6.81 & 6.40 & 5.43 & 8.11 & 5.09 & 5.06 & 5.27 \\
\hline Back home & 46.08 & 43.70 & 46.10 & 42.87 & 46.89 & 46.35 & 46.17 & 46.57 \\
\hline Business trips & 3.34 & 3.59 & 2.80 & 6.68 & 2.41 & 1.59 & 2.63 & 1.66 \\
\hline Return trips & 1.82 & 2.74 & 1.90 & 2.19 & 1.02 & 1.03 & 0.09 & 0.04 \\
\hline Shopping & - & 7.61 & 8.30 & 6.06 & 4.58 & 11.80 & 9.02 & 8.02 \\
\hline Daily routines & 10.14 & 9.11 & 13.00 & 11.78 & 9.57 & 8.74 & 14.99 & 14.58 \\
\hline Other trips & 0.58 & 3.75 & 3.30 & 1.71 & 1.11 & 6.33 & 0.28 & 1.61 \\
\hline
\end{tabular}

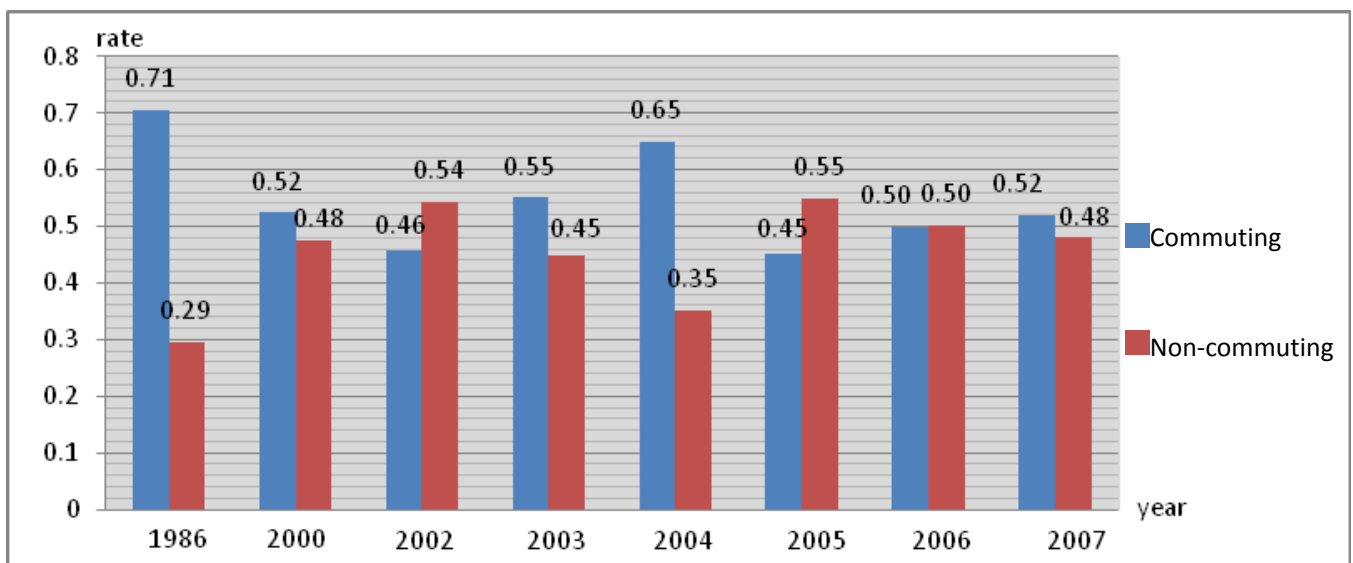

Fig. 4. Rate of commuting and non-commuting travels in Beijing (1986-2007)

Table 5 only presents the raw data. We should remove disturbing vague figures and merge figures that belong to the same main category. The concept of trips back home and returning trips is obscure, since these two types of trips cannot be clearly distinguished, and it is possible to say that trips back home and return trips should be in the same category. Theoretically speaking, return trips are generated by other types of trips, so we need to find out the proportion of different returning trips initiated by discrepant trip purposes respectively, and add the number 
of a specific kind of return trips to the figure of the pertinent trip purpose. However, this measure is neither practical nor necessary. Instead, we could remove figures of trips back home and returning trips, and only consider the start offs. Besides, in order to study the trends discrepancy between work and non-commuting travel, we can merge the figures of commuting and schooling together as the newly defined commuting travel. Simultaneously, we can combine business trips, shopping, daily routines and other trips together as
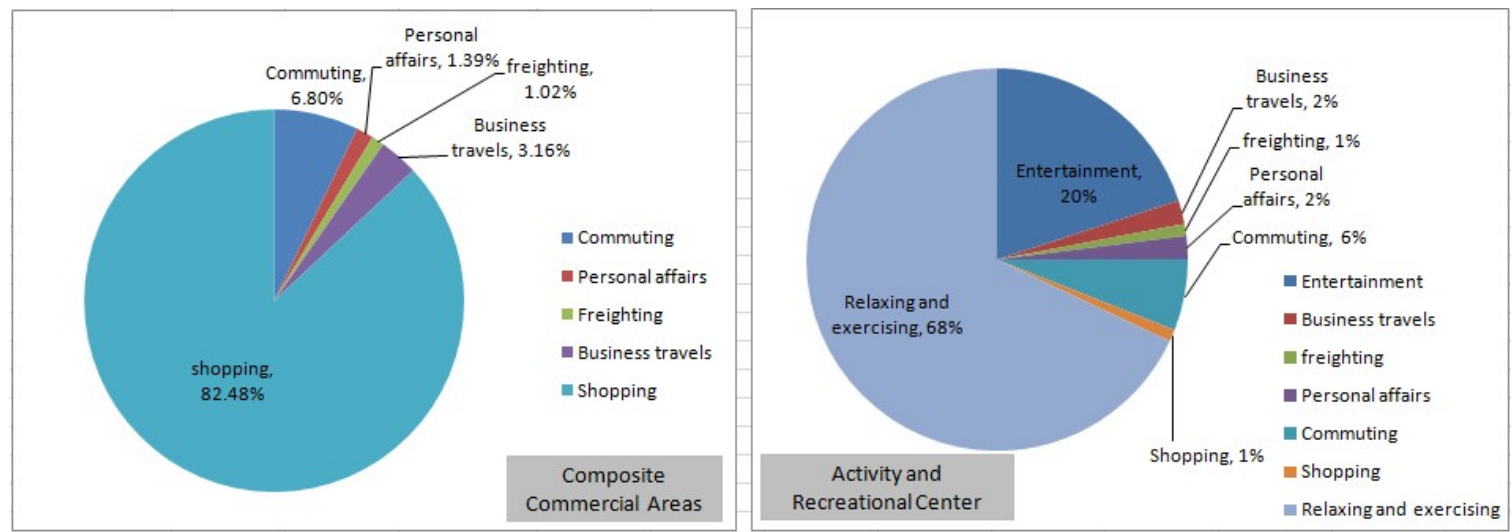

Fig. 5. Trip purpose distribution for people arriving at commercial and recreational areas in Beijing (2007) Source: Report on traveler-inviting sites of the third integrating investigation in Beijing transportation

non-commuting trips shown in Fig.4.

Fig.4 shows that in 1986, trips generated by commuting and school substantially surpassed that for non-commuting purposes (71\% compared to 29\%). By contrast, during the period between 2000 and 2007, the change toward the opposite is conspicuous. Except for
2003 and 2004, when there was a relative larger gap between the ratio of these work and non-commuting travels, the proportion of work and non-commuting travel in other years almost equaled. And it is noticeable that in 2005, the ratio of non-commuting travel (55\%) even surpasses that of work travel

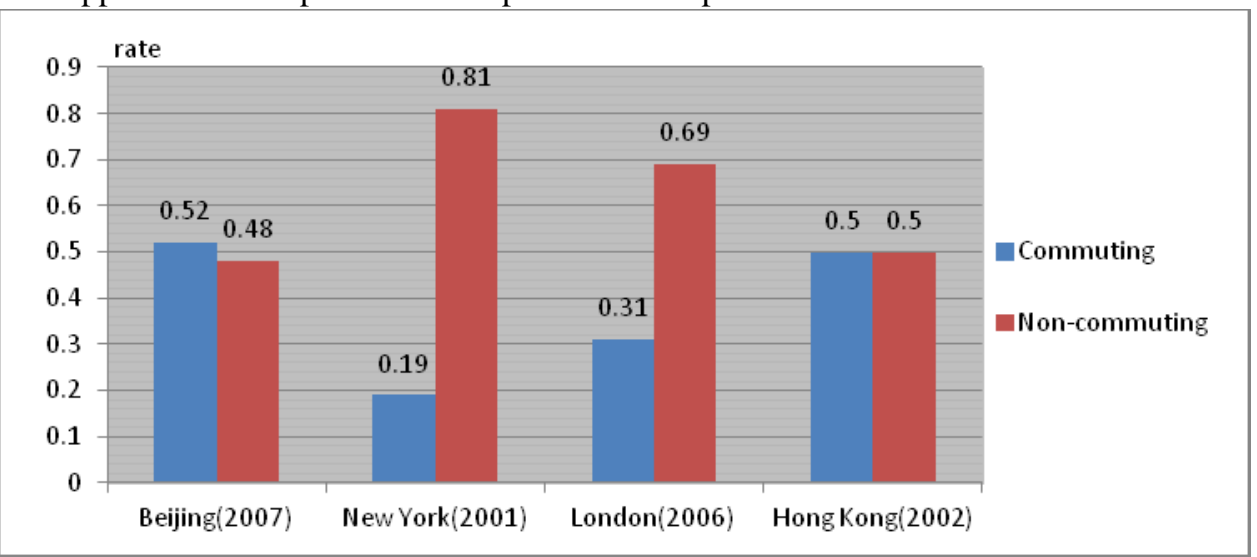

Fig. 6. Rate of commuting and non-commuting travels in Beijing, New York, London and Hong Kong

Source: Annual Report of Beijing Transport Development (2008). National Household Travel Survey New York Add-On (2001); London Travel Report (2007). Hong Kong Travel Characteristics Survey (2002).

(45\%). The inconsistency of figures in 2003 and 2004 could be interpreted by the data shown in Table 1. In 2003, the survey was carried in the communities of Huilongguan, Qinghe, Tiantongyuan, and Beiyuan, and these communities are rather far away from down town areas and recreational centers. A large proportion of residents living in these regions is white collar workers, the commuting is the main trip purpose. As shown in Fig.5, non-commuting travels are usually generated in commercial and recreational areas ${ }^{22}$, and the lack of such areas in proximity makes it quite unlikely for residents to take frequent non-commuting travels. Similarly, in 2004, 
the survey was conducted in a selected part of Beijing, thus the data acquired may not accurately represent the whole picture of the city in the year.

Most significantly, the proportions of non-commuting travel even surpassed those of commuting travel in 2002 and 2005. Since the total amount of commuting travel is quite inflexible, a decline in the ratio of trips related to work and school means an increase in the total number of non-commuting travel, indicating an ascend in the entire number of trips. This could be supported by the data in Fig. 1 and Fig.2, showing that 2002 and 2005 witnessed the largest number of trips per capita per day.

Meanwhile, considering the growing demand of traffic service due to the increasing population in Beijing, it is evident that the number of non-commuting trips will grow to a higher level.
It appears that residents in Beijing have changed their travel habits in recent years. The changes may be attributed to the increased wealth in urban areas. Trips not related to commuting have already enjoyed a higher percentage, and initiatives need to be taken to meet the growing requirements.

It can be inferred that the percentage of non-commuting travel correlates with the level of prosperity in a city. By analyzing the distribution of commuting and non-commuting travel in New York, London and Hong Kong (see Fig.6), this trend seems justifiable. In New York, the proportion of non-commuting travel is tremendously higher than that of commuting travel, $81 \%$ to $19 \%$. In London, the ratios of non-commuting and commuting travel are $69 \%$ and $31 \%$ respectively. In Hong Kong, the two types of travel take up the same percentage. In contrast,

Table 6: Travel modes in Beijing from 1986 to 2007 (\%)

\begin{tabular}{lllllllll}
\hline Travel Mode & 1986 & 2000 & 2002 & 2003 & 2004 & 2005 & 2006 & 2007 \\
\hline Public transportation & 28.54 & 35.27 & 34.40 & 33.70 & 27.06 & 37.40 & 38.30 & 42.20 \\
Car & 5.04 & 26.23 & 12.30 & 35.90 & 30.30 & 32.30 & 34.00 & 34.80 \\
Bike & 62.65 & 38.49 & 50.10 & 16.60 & 42.50 & 30.30 & 27.70 & 23.00 \\
Others & 3.77 & 0.00 & 3.20 & 14.30 & 0.00 & 0.00 & 0.00 & 0.00 \\
Total & 100 & 100 & 100 & 100 & 100 & 100 & 100 & 100 \\
\hline
\end{tabular}

non-commuting travel in Beijing accounts for $48 \%$, relatively lower than commuting travel. Although the data is not acquired in the same year, it is generally acknowledged that in such highly developed cities as New York, London and Hong Kong, the travel modes and structure would remain relatively stable in a long time span, which gives us adequate reasons to overlook possible disparities if the surveys were conducted at a different time. And it is reasonable to predict that although the rate of non-commuting travel in Beijing is the lowest compared with London, New York and Hong Kong, it is very likely to grow in the coming years due to the rapid economic

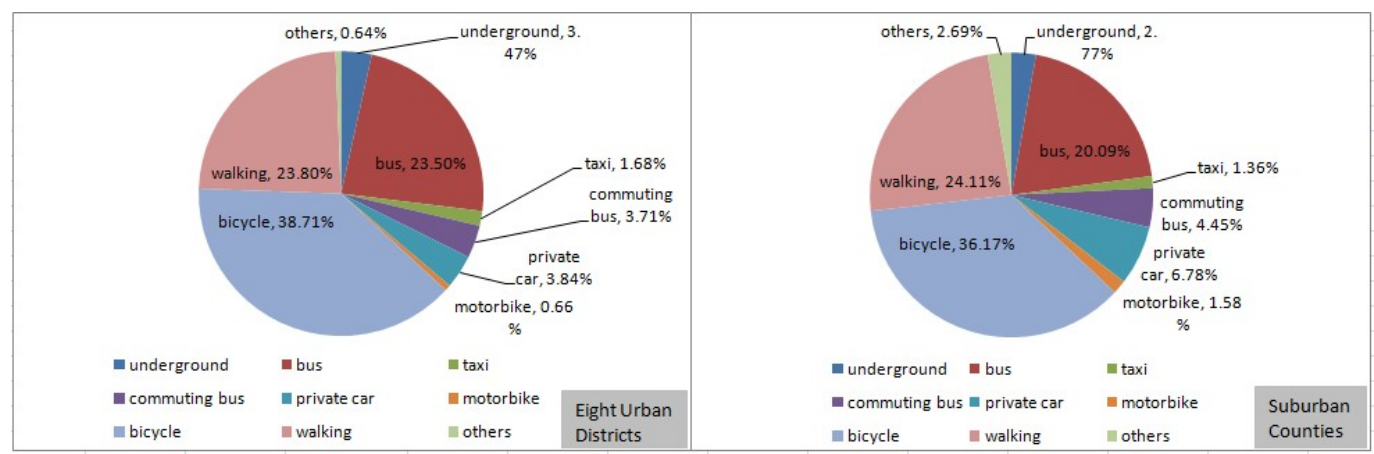

Fig. 7. Proportions of travel modes for families with a car ownership in Beijing (2005) Source: Annual Report of Beijing Transport Development (2005) 
development. The ratio of non-commuting travel for Beijing residents would gradually increase to a much higher level in the near future ${ }^{1}$.

\subsection{Travel Mode}

The structure of travel mode is commonly defined as the proportions of each mode chosen for the trips. It could reflect the level of transportation development and people's attitudes toward travel modes. Table 6 presents the distribution of various travel modes from 1986 to 2007 in Beijing. In Table 6, "public transportation” includes underground, local bus, mini bus and commuting bus; "Car" includes private cars, taxis and company cars; "bike" includes motorbikes and bicycles; "Others" includes walking and other means of transportation not mentioned above.

Table 6 shows that during the past 20 years, the percentage of travel by bicycles dropped from $62.65 \%$ in 1986 to $23 \%$ in 2007. Meanwhile, the percentage of public transportation increased enormously from 28.54\% in 1986 to $42.40 \%$ in 2007 . It is also noticeable that travel by private cars became more and more popular, with a dramatic increase from only $5.04 \%$ in 1986 to $34.8 \%$ in 2007, and the increase is approximately six-fold.

Table 6 provides clear information of the trends of changes on the rate of different travel modes from 1986 to 2007. However, it classifies travel modes in a relative simple way, and it doesn't describe proportions of travel modes in different kinds of families. Fig.7 and Fig.8 help complete the deficient information. Fig.6 is the distribution of travel modes of families with a car ownership in 2004, and Fig.7 presents the ratios of travel modes of families without a private car in the same year. It is clearly illustrated in the charts that an overwhelmingly majority of car-owner families would choose to drive when taking a trip, with $55.08 \%$ and $49.16 \%$ respectively for families in eight urban districts and suburb areas. Meanwhile, families without cars would take bicycle as their priority, with $38.71 \%$ and $36.17 \%$ respectively for families in the two types of areas stated above. It can be easily inferred that public transportation has not yet gained enough popularity. Even for a family without a car, the proportion of trips taken by underground and bas is only approximately $25 \%$. In order to propagate the concept of "public transport oriented

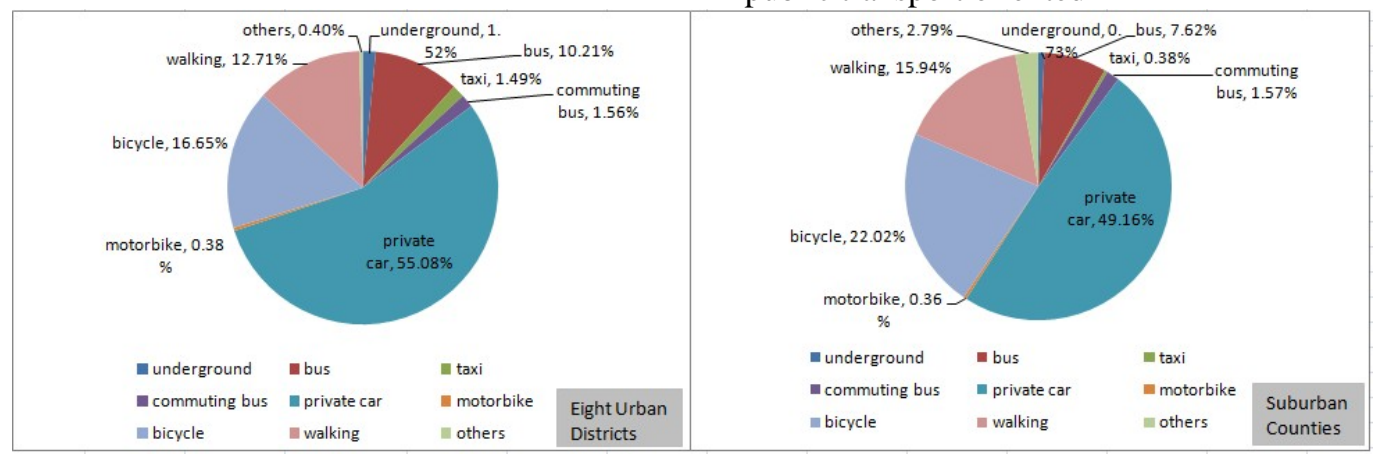

Fig. 8. Proportions of travel modes for families without a car ownership in Beijing (2005) Source: Annual Report of Beijing Transport Development (2005)

city”, the improvement of current public transport system is in demand to rival private cars and bicycles in their convenience and level of comfort.

\subsection{Travel modes of commuting and non-commuting travels}

Table 7 presents the distribution of different traffic modes for commuting and non-commuting travels in Beijing (2005). It is easy to find that for commuting travel, percentages of public transportation, private cars and bicycles are almost similar, and the figure of private cars slightly surpass the other two modes. However, for non-commuting travel, the percentages of public transportation and bicycles are relatively higher than private cars, with 38\%, 34\% and $24.63 \%$ respectively. However, we cannot safely draw the conclusion that public transportation system is sufficiently developed to satisfy the commuting and non-commuting needs simply by its proportion. References should be put forward to better understand the status of commuting and non-commuting travels in Beijing. 
Table 7: Travel modes for commuting and non-commuting travel in Beijing(2005)（\%）

\begin{tabular}{|c|c|c|c|c|c|}
\hline & Public transport & Private cars & bicycles & Others & Total \\
\hline Commuting travel & 30.94 & 33.96 & 30.49 & 4.61 & 100 \\
\hline Non-commuting travel & 38.41 & 24.63 & 33.78 & 3.18 & 100 \\
\hline
\end{tabular}

Source: Annual Report of Beijing Transport Development (2006)

Compared with the data of New York(2001) and Hong York and Hong Kong, accounting for 52\% and 94\% Kong(2002) (shown in Fig.9), it is clear that for commuting travel, public transportation plays the most respectively. Comparatively, the percentage of commuting travel by public transportation in Beijing is far from these significant role among all the travelling modes in the New levels (31\%).
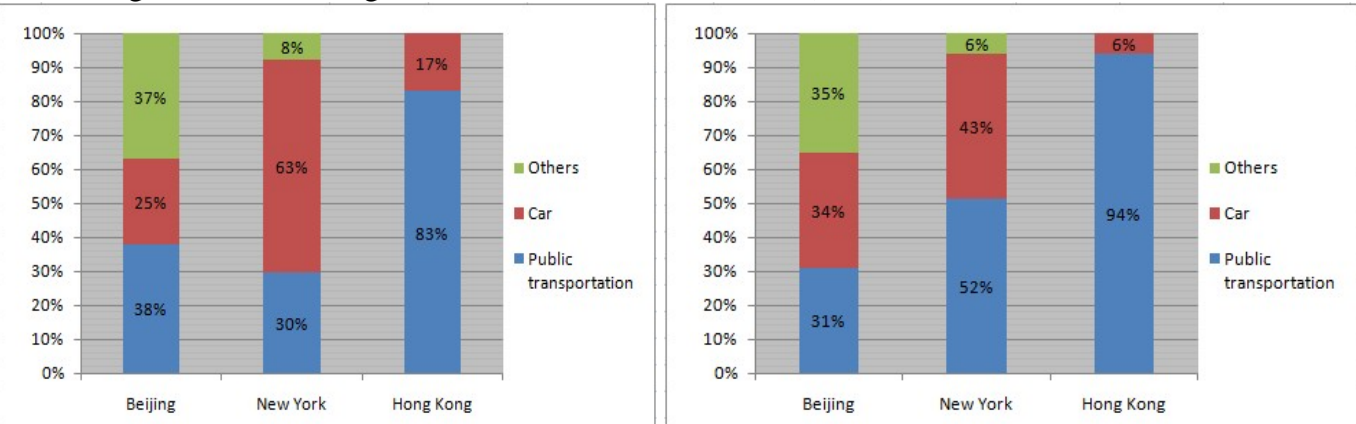

Fig. 9. Proportions of travel modes for commuting travels (left) and non-commuting travels (right) in Beijing, New York and Hong Kong

Source: Annual Report of Beijing Transport Development (2006); National Household Travel Survey New York Add-On (2001); Hong Kong Travel Characteristics Survey (2002)

As for non-commuting travel, public transportation is overwhelmingly overrepresented in Hong Kong, accounting for $83 \%$ of all the non-commuting travels, while in New York, private cars enjoy a majority of $63 \%$ among all the modes as opposed to $30 \%$ for public transportation. It should be considered that in the United States, especially in metropolis as New York, the percentage of people with car ownership is considerable. And people in the US are more used to driving a car

Table 8: Trip purposes and travel modes in Beijing (2005) (return trips neglected) (\%)

\begin{tabular}{llllll}
\hline Trip purpose & Private cars & Taxis & Public transport & Commuting bus & Bicycle \\
\hline Dining out & 1.3 & 1.6 & 0.3 & 0.1 & 0.3 \\
Business trips & 9.0 & 10.4 & 2.0 & 0.7 & 1.5 \\
Shopping & 6.4 & 6.1 & 9.0 & 6.9 & 13.2 \\
Sending goods/services & 13.5 & 5.2 & 2.4 & 1.0 & 6.8 \\
Seeing a doctor & 1.3 & 7.5 & 1.6 & 0.3 & 1.0 \\
Other purposes & 2.3 & 4.5 & 2.0 & 1.4 & 1.9 \\
Commuting & 29.4 & 27.7 & 27.8 & 38.8 & 29.3 \\
Schooling & 2.5 & 2.9 & 6.1 & 7.2 & 7.4 \\
Visiting friends & 3.6 & 5.6 & 4.1 & 0.2 & 1.6 \\
Entertainment & 0.5 & 1.3 & 0.8 & 0.2 & 0.5 \\
Return from work & 27.9 & 23.6 & 32.2 & 36.0 & 26.6 \\
Return from school & 2.0 & 2.8 & 8.5 & 7.1 & 7.3 \\
Relaxation & 0.5 & 0.8 & 3.0 & 0.0 & 2.6 \\
Total & 100 & 100 & 100 & 100 & 100 \\
\hline
\end{tabular}

compared to people in Beijing. Meanwhile, since popularization of public transportation is strongly advocated in Beijing, and the number of car ownership is to be controlled, it is highly recommended that the rates of 
travel modes in Hong Kong could be a good example for Beijing. We are already aware of the fact that the rate of public transportation in commuting travel is to be increased in the past few years. Considering the situation in Hong Kong, where public transportation takes up 83\% of non-commuting travels in 2002, it seems that its percentage in non-commuting travel still needs to get enhanced.

It can be inferred from the disparity between Beijing and Hong Kong that in order to enhance the percentage of public transportation in the coming years, not only should city planners consider propagating public transportation for commuting travel but as an important ancillary public transportation for non-commuting travel should also gain its preponderance.

\subsection{The distribution of trip purposes for public transportation}

Table 8 illustrates the distribution of different trip purposes for various means of transportation in Beijing (2005). And Fig.10 visually presents information in Table 8. It is showed that among all the purposes of non-commuting travel, shopping appears to be the main reasons for people to choose public transportation. Private cars are most likely to be used for business trips, and visiting friends. Taxis are most likely to be used for business trips and seeing a doctor

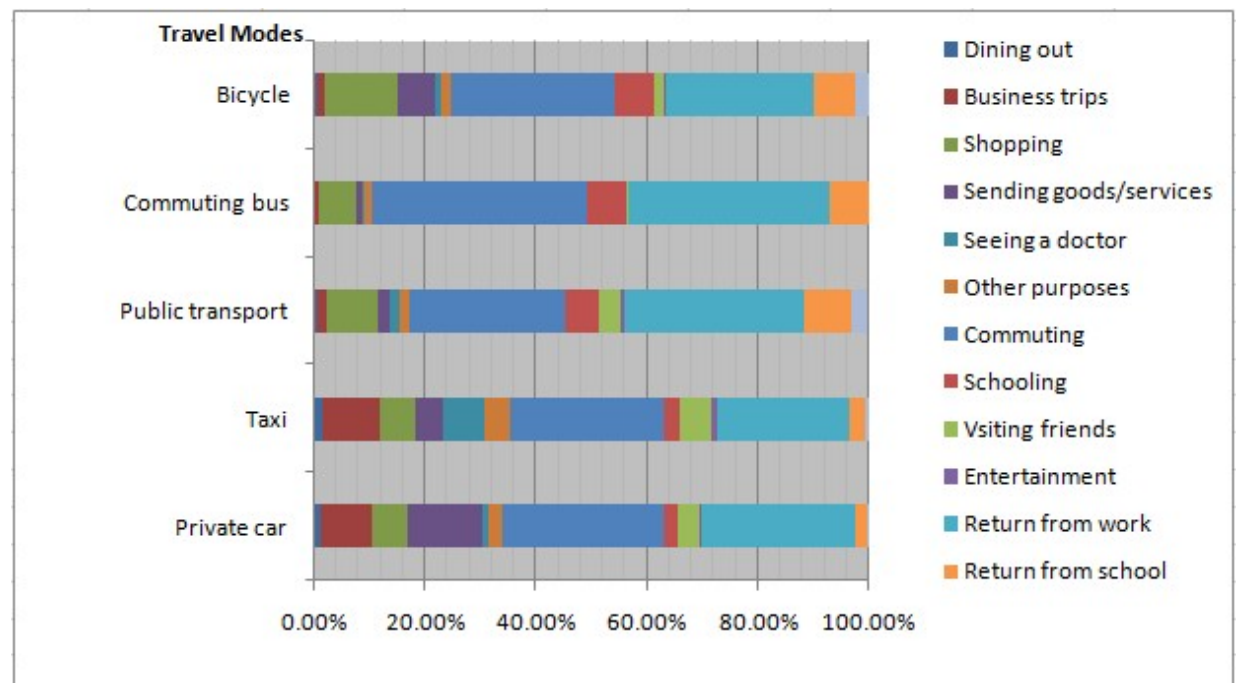

Fig. 10. Trip purposes and travel modes in Beijing (2005) (return trips neglected)(\%) Source: Annual Report of Beijing Transport Development (2006)

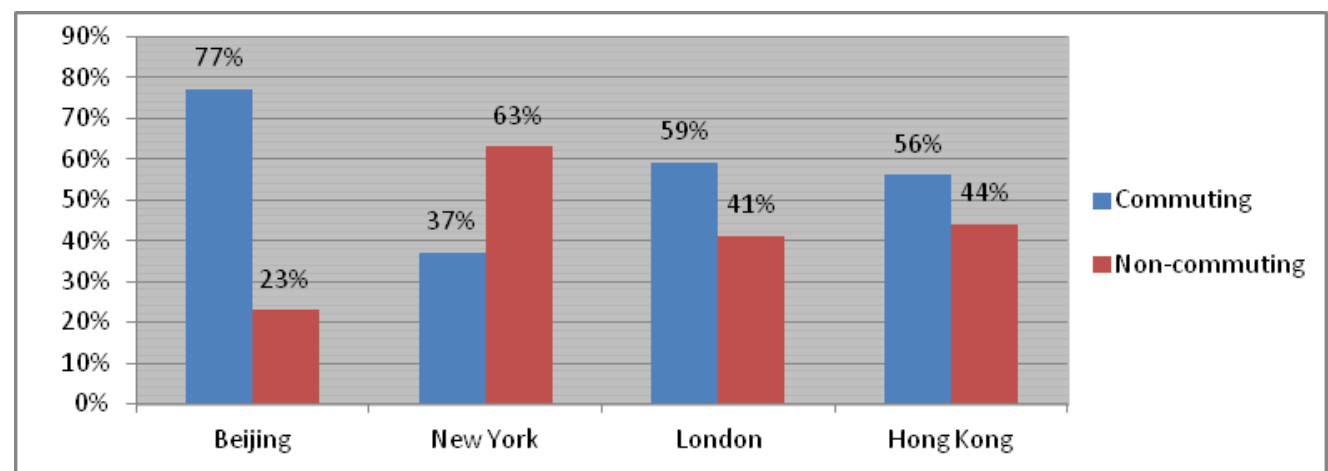

Fig. 11. Commuting and non-commuting travels in different cities

Source: Annual Report of Beijing Transport Development (2006); National Household Travel Survey New York Add-On (2001); London Travel Report (2007); Hong Kong Travel Characteristics Survey (2002) 
As compared to the data of New York(2001), London(2006) and Hong Kong(2002) (Fig.11), we could find that except for New York, where the percentage of non-commuting travels (63\%) by public transportation surpasses that of commuting travel (37\%), all the investigated cities report a higher rate of commuting travels by public transportation. However, both in London and Hong Kong the gaps between the percentages of work and non-commuting travels by public transportation are small, while in Beijing a large discrepancy exists between work and non-commuting travels by public transportation (77\% compared to 23\%).

The data reveals a significant general situation about the percentage of non-commuting travel in a metropolis with a maturely developed public transportation system, that is, non-commuting travel also relies on public transportation on a large scale. It implies that the rate of non-commuting travel by public transportation in Beijing should be increased; the public transportation system in Beijing can be more effective and more widely used. At the same time, service improving should be prioritized to encourage passengers for non-commuting reasons.

\section{Concluding remarks and future work}

In sum, the above analyses have determined the fundamental travel demands of Beijing residents and identified the changing patterns of commuting and non-commuting travels in Beijing. Statistical data of Beijing is studied regarding number of trips, trip purpose, travel modes for commuting and non-commuting travels and distribution of trip purposes for public transportation. These patterns have been compared with highly developed cities such as London, New York and Hong Kong. Several important conclusions can be drawn from this exploratory study.

The first important conclusion is that the travel demand of Beijing residents has considerably grown in the past twenty years. The per capita number of trip in Beijing has experienced a dramatic increase, from 1.61 times per day in 1986 to 2.53 in 2007. And the increasing rate is 0.04 times per day, which substantially surpasses the rate of cities in developed countries. The social changes and economic development in the past twenty years have enriched the life of Beijing residents, and it has led to greater travel demands and placed a pressure on Beijing's transportation system. Meanwhile, as the size of population in Beijing is growing both in terms of permanent residents and in terms of the floating population, the travel demands of Beijing residents would continue to grow and should be regarded as a serious challenge to the transportation system.

The second important conclusion is that the past twenty years witnessed a rapid increase of the rate of non-commuting travel. This shows that people's leisure time has increased. People can spend more time on entertainment and social activities to enrich their life. Comparing the situation of Beijing with New York, London and Hong Kong, we could conclude that in a more developed city the non-commuting travel needs is more likely to surpass that of commuting travel. Thus, we can anticipate that in a developing city like Beijing the need for non-commuting travel will continue to increase and play a more significant part. As a result, the distribution of time and space in transportation system would be more flexible and unpredictable, and this would be more challenging than satisfying the demands of commuting travel, which is more fixed and predictable.

Thirdly, it is illustrated in the distribution of travel modes that public transportation in Beijing should gain a higher proportion, comparing with exploding numbers of private car in recent years. Comparative analysis of corresponding data of Hong Kong reveals that increasing the proportion of public transportation in non-commuting travel is as well substantial although that in commuting travel has long been the issue considered. Meanwhile, as support data proportions of commuting and non-commuting travels by public transportation in Beijing, New York, London and Hong Kong are studied, which pointed out that the rate of non-commuting travel needs to be increased, and public transportation service for this continuously increasing type of trip purpose leaves much room for development.

It should be mentioned that the data presented in this paper is not the most recent resource, and there is a 3 to 4 year gap between the comparison data of Beijing and other cities. In order to make more precise and up to date analysis, the most recent data and comparison based on data of the same year are recommended. Moreover, this paper classifies trip purposes in a relative simple way, and more detailed classification is demanded to deeply investigate what kind of trip purpose is currently least satisfied by the public transportation system, thus presenting more crucial and direct suggestions to optimize public transportation nowadays. It is also recommended in 
the future work to take the influence of policies related to public transportation into account, such as the policy of "public transportation oriented city" and ticket price policy, which is substantial for understanding the travel characteristics of citizens.

\section{References}

1. Research Group of Travel Demand of Beijing Social Economic Research Agency, The trend of social and economic growth in Beijing from 2003 to 2020 (In Chinese), Technical Report (Beijing Social Economic Research Agency, 2003).

2. H. Bubb, Traffic safety through driver assistance and intelligence. International Journal of Computational Intelligence Systems. 4(3)(2011)287-296.

3. R. Cervero, The transit metropolis (Island Press.Washiton, D.C. • Covelo, CA, 1998) .

4. I. Lopez-Yanez, A. J. Arguelles-Cruz, O. Camacho-Nieto, C. Yanez-Marquez, Polluants time-series prediction using the gamma classifier. International Journal of Computational Intelligence Systems. 4(4)(2011)680-711.

5. Chinese Research Agency of Urban Planning, China urban public transportation development report (2007) (In Chinese), Technical Report(2008).

6. X. M. Lu, Metropolis transport strategy (In Chinese) (China Architecture \& Building Press, Beijing, 2006).

7. A. N. Cohen, Public transportation is not going to work: non-commuting travel markets for the future of mass transit, Master Thesis (Massachusetts Institute of Technology, 2004).

8. Beijing Transportation Research Center, Annual Report of Beijing Transport Development (In Chinese), Technical Report(1986).

9. Beijing Transportation Research Center, Annual Report of Beijing Transport Development (In Chinese), Technical Report(2000).

10. Beijing Transportation Research Center, Annual Report of Beijing Transport Development (In Chinese), Technical Report(2002).

11. Beijing Transportation Research Center, Annual Report of Beijing Transport Development (In Chinese), Technical Report(2003).

12. Beijing Transportation Research Center, Annual Report of Beijing Transport Development (In Chinese), Technical Report(2004).

13. Beijing Transportation Research Center, Annual Report of Beijing Transport Development (In Chinese), Technical Report(2005).

14. Beijing Transportation Research Center, Annual Report of Beijing Transport Development (In Chinese), Technical Report(2006).
15. Beijing Transportation Research Center, Annual Report of Beijing Transport Development (In Chinese), Technical Report(2007).

16. Arup Wilbur Smith Associate, Travel characteristics survey 2002 final report, Technical Report (Transport Department, 2003).

17. Oak Ridge National Laboratory, 1995 National household travel survey, New York add-on, Technical Report (UT-Battelle, 1997).

18. Oak Ridge National Laboratory, and MacroSys Research and Technology, 2001 National household travel survey, New York add-on, Technical Report (UT-Battelle, 2004).

19. Great Briton Department of Transportation, Transport statistics for metropolitan areas (The Stationery Office, Norwich, 2000).

20. Steer Davies Gleave, London travel report 2007, Technical Report (Transport for London, 2010).

21. Beijing Statistical Information Center, Beijing statistical yearbook (In Chinese), Technical Report (2010).

22. Beijing Transportation Committee, and Beijing Transportation Research Center, Report on traveler-inviting sites of the third integrating investigation in Beijing transportation (In Chinese), Technical Report (2007). 